Original Article

\title{
Comparison of low frequency repetitive transcranial magnetic stimulation parameters on motor cortex excitability in normal subjects
}

\author{
Lara M. Schrader ${ }^{a, *}$, Sima Sadeghinejad ${ }^{a, c}$, Jalleh Sadeghinejad ${ }^{a, c}$, \\ Mouses Kazanchyan ${ }^{a, c}$, Lisa Koski ${ }^{b}$, John M. Stern ${ }^{a}$, Allan D. Wu ${ }^{a}$, \\ Marco Iacoboni ${ }^{c}$, Marc R. Nuwer ${ }^{a}$ \\ ${ }^{a}$ David Geffen School of Medicine at UCLA, Department of Neurology, Los Angeles, CA 90095, USA \\ ${ }^{\mathrm{b}}$ McGill University, Department of Medicine, Montreal, Quebec, Canada \\ ${ }^{\mathrm{c}}$ Ahmanson-Lovelace Brain Mapping Center, Brain Research Center, David Geffen School of Medicine at UCLA, \\ Department of Psychiatry and Biobehavioral Sciences, Los Angeles, CA 90095, USA
}

\section{A R T I C L E I N F O}

\section{Article history:}

Received 2 January 2015

Accepted 22 October 2015

Available online 23 April 2016

Keywords:

Low frequency repetitive

transcranial magnetic stimulation

Stimulation parameters

Cortical excitability

Brain stimulation

\begin{abstract}
A B S T R A C T
Background/objectives: Optimal low frequency repetitive transcranial magnetic stimulation (LF-rTMS) parameters for treating epilepsy and other brain disorders are unknown. To address this question, a systematic study of the effects of LF-rTMS frequency and intensity on cortical excitability was performed.

Methods: Using a four-period crossover design, subjects were scheduled for four LF-rTMS sessions that were at least four weeks apart. LF-rTMS was delivered as 900 pulses directed at primary motor cortex using four protocols: $0.5 \mathrm{~Hz}$ at $90 \%$ resting motor threshold (RMT), $0.5 \mathrm{~Hz}$ at $110 \% \mathrm{RMT}, 1 \mathrm{~Hz}$ at $90 \% \mathrm{RMT}$, and $1 \mathrm{~Hz}$ at $110 \%$ RMT. Motor evoked potential (MEP) amplitude, resting motor threshold (RMT), and cortical silent period (CSP) were measured before, immediately after, and $60 \mathrm{~min}$ after LF-rTMS. Each of the four protocols was analyzed separately to compare baseline measurements to those after LF-rTMS.

Results: None of the four LF-rTMS protocols produced a trend or significant change in MEP amplitude, RMT, or CSP.

Conclusion: The lack of significant effect from the four LF-rTMS protocols indicates that none produced evidence for alteration of cortical excitability. The direct comparison of four LFrTMS protocols is distinct to this investigation, as most similar studies were exploratory and studied only one or two protocols. The negative result relates only to the methods used in this investigation and does not indicate that LF-rTMS does not alter cortical excitability with other parameters. These results may be useful when designing additional investigations into the effect of LF-rTMS on epilepsy, other disorders, and cortical excitability.

(C) 2016 Indian Epilepsy Society. Published by Elsevier, a division of Reed Elsevier India,
\end{abstract} Pvt. Ltd. All rights reserved.

\footnotetext{
* Corresponding author at: 710 Westwood Plaza, Room 1-194, Los Angeles, CA 90095, USA. Tel.: +1 3102063093 ; fax: +1 3102671157.

E-mail address: lschrader@mednet.ucla.edu (L.M. Schrader).

http://dx.doi.org/10.1016/j.ijep.2016.02.003

2213-6320/C 2016 Indian Epilepsy Society. Published by Elsevier, a division of Reed Elsevier India, Pvt. Ltd. All rights reserved.
} 


\section{Introduction}

Transcranial magnetic stimulation (TMS) is a non-invasive technique for brain stimulation that can measure the state of cortical excitability and can also be used to alter the excitability. Altering cortical excitability occurs when the TMS pulses are delivered repetitively (rTMS), and the excitability is decreased in the stimulated region if the pulses rate is $1 \mathrm{~Hz}$ or less (low frequency). rTMS has been used as a treatment for several brain disorders, but its use in epilepsy is not well established because of mixed results. ${ }^{1-6}$ The development of LF-rTMS for epilepsy will require additional understanding of its effects and implementation.

Optimal LF-rTMS parameters for altering cortical excitability are unknown, and this has impeded clinical research into LF-rTMS as a treatment for epilepsy, which is known to have abnormality of cortical excitability. ${ }^{7}$ Because several studies have reported significant and discordant findings from varying LF-rTMS stimulation parameters, we sought to address this issue by systematically examining the effects of LF-rTMS frequency and intensity using a four-period crossover design. $^{8-12}$ The primary outcome measures were assessed immediately before and immediately after LF-rTMS. A secondary outcome measure was duration of effect, as assessed by measuring RMT, CSP, and MEP amplitude 60 min after LFrTMS. The results of this investigation are intended to be useful when planning LF-rTMS treatment trials by providing a basis for selecting stimulation parameters.

\section{Methods}

Thirteen healthy subjects (10 women and 3 men, age 19-37 years) participated. Eleven were right-handed, and two were left-handed as assessed by the Edinburgh Handedness Inventory. ${ }^{13}$ The University of California, Los Angeles (UCLA) Institutional Review Board approved the protocol, and informed consent was obtained from all subjects. A boardcertified neurologist performed a neurological exam before and after each session. Subjects were questioned at the end of each session about adverse effects. To decrease variability, the same examiner (SS or JS) held the coil for all four sessions that each individual subject underwent. ${ }^{14,15}$

\subsection{Cortical excitability measurements using single-pulse TMS}

To minimize the chance of finding a false positive result due to multiple testing, analysis was restricted to three primary outcome measures: change in resting motor threshold (RMT), cortical silent period (CSP), and motor evoked potential (MEP) amplitude. Cortical excitability measurements were obtained using a Cadwell High Speed Magnetic Stimulator (Kennewick, WA, USA) and a $9 \mathrm{~cm}$ focal point coil. The stimulator produces single cosine wave pulses of approximately 200 microseconds duration. The round portion of the coil was held flush with the scalp such that the coil's tip was pointed anteriorly and elevated off the scalp. The "hot spot" for the dominant hemisphere was found by starting at the vertex and exploring the region just lateral and anterior to the vertex to locate the coil placement that reliably produced MEPs amplitudes of the highest amplitude. A clockwise current was used to stimulate the right hemisphere, while a counterclockwise current was used for the left. EMG signals were recorded using metal disk electrodes taped to the muscle belly and tendon of the dominant hand's first dorsal interosseous (FDI) muscle. A filter bandpass of 1-1000 $\mathrm{Hz}$ and sampling rate of $1000 \mathrm{~Hz}$ were used. The data were digitally displayed and stored in 500 millisecond samples for later analysis (Labview, National Instruments). To assess muscle activity preceding the TMS pulse, 100 milliseconds of EMG was recorded prior to the TMS pulse in addition to 400 milliseconds after each pulse. Single-pulse TMS was administered using pulses separated by 5-10 seconds.

Each session began with single-pulse TMS that was applied to the dominant hemisphere to determine the scalp location producing the most reliable and highest amplitude MEP for the FDI (also known as the "hot spot"). This scalp location that was used for all cortical excitability measurements for the remainder of the session. The location and orientation of the coil with respect to the head when the hot spot was found were carefully marked on a tight-fitting elastic swim cap worn by the subject.

For each subject, four separate TMS sessions were scheduled at least four weeks apart. Within each session, dominant hemisphere cortical excitability was measured: (1) before LF-rTMS (Pre), (2) immediately after LF-rTMS (Post), and (3) $60 \mathrm{~min}$ after LF-rTMS (60 min). Cortical excitability was evaluated using RMT, MEP amplitude, and CSP, measured in fixed order.

To determine the RMT, the TMS intensity was reduced in step-wise decrements of $2 \%$ to find the intensity that produced an MEP of at least $50 \mu \mathrm{V}$ peak-to-peak amplitude for at least 5 of 10 stimulations. If the last $2 \%$ decrement was below RMT, the stimulator output was increased by $1 \%$ to determine if the intermediate intensity was the RMT.

To determine the MEP amplitude, 20 MEPs were acquired from the FDI with the muscle at rest using an intensity of $120 \%$ RMT. The average amplitude of the 20 MEPs was calculated. If muscle activity was present during the $100 \mathrm{~ms}$ preceding the TMS pulse, that MEP was discarded and an additional MEP was acquired in its place.

To determine the CSP duration, ten CSPs were recorded, and the average duration of the 10 CSPs was calculated. Each CSP was obtained using an intensity of $120 \%$ RMT while the dominant hand FDI was isometrically contracting at $10 \%$ maximum voluntary contraction. Prior to administering TMS pulses for CSP acquisition, subjects pinched a partially inflated sphygmomanometer with maximal force between the index finger and thumb with the index finger abducting against the thumb to activate the FDI. The sphygmomanometer reading for maximal force was recorded. During the CSP acquisition, subjects watched the sphygmomanometer dial and held the pinch at $10 \%$ of maximal force.

The MEP and CSP data were analyzed off-line with a modular MATLAB (Mathworks, MA) software data analysis tool, dataWizard (version 0.5.1, A.D.W., UCLA) and individually verified by visual inspection by the same investigator. The MEP 
amplitude for each tracing was the voltage difference between the peak and trough of each MEP. The CSP duration was measured from the MEP onset to the sustained reappearance of rectified, integrated EMG activity of at least $20 \mu \mathrm{V}$ amplitude.

\subsection{LF-rTMS}

LF-rTMS was performed using a Magstim Super Rapid biphasic stimulator (current induced postero-anterior followed by antero-posterior) with a rise of time of approximately $80 \mu \mathrm{s}$ and a total pulse duration of $250 \mu$ s via an air-cooled figure-of8 coil with an outside diameter of $7 \mathrm{~cm}$ per wing (The Magstim Company Ltd, Wales, UK). The point of intersection of the figure-of-8 coil was placed against the skull, and the coil was held at a 45-degree angle to the sagittal plane with the handle oriented posterolaterally. ${ }^{16}$

Using a four-period crossover (Latin square) design, subjects underwent four LF-rTMS sessions at least four weeks apart. At each session, 900 pulses were directed at the dominant primary motor cortex using one of four LF-rTMS protocols: A) $0.5 \mathrm{~Hz}$ at 90\% RMT, B) $0.5 \mathrm{~Hz}$ at 110\% RMT, C) $1 \mathrm{~Hz}$ at $90 \% \mathrm{RMT}$, and D) $1 \mathrm{~Hz}$ at $110 \%$ RMT. Subjects were assigned to one of four groups. The group to which the subject was assigned determined the order in which the individual underwent the different LF-rTMS protocols, A-D:

Group 1: First A, then B, then C, then D.

Group 2: First $B$, then $C$, then $D$, then $A$.

Group 3: First $C$, then $D$, then $A$, then $B$.

Group 4: First D, then A, then B, then $C$.

The first subject was assigned to Group 1, the second to Group 2, and so on. Because of dropouts, some subjects were assigned to a group out of order in attempt to balance the number of subjects in each group.

\subsection{Statistical analysis}

Values for RMT, MEP amplitude, and CSP were obtained at baseline (Pre), immediately after LF-rTMS (Post) and $60 \mathrm{~min}$ after LF-rTMS (60 min). For analysis of data from all four periods, each of the four LF-rTMS protocols was analyzed separately using paired t-tests to determine whether a significant change in RMT, CSP, and MEP amplitude occurred immediately after (Pre compared to Post) or 60 min after (Pre compared to $60 \mathrm{~min}$ ) LF-rTMS. Due to a high drop out rate, analyses comparing four different LF-rTMS protocols were not done to avoid unknown biases from missing data.

\section{Results}

\subsection{Effect of LF-rTMS on RMT, MEP amplitude, and CSP}

Of 13 subjects, seven completed all four sessions, one completed three, one completed two, and four completed one. Nine underwent protocol $A$, ten underwent $B$, nine underwent $C$, and nine underwent $D$, so each protocol had results for at least 9 subjects. No LF-rTMS protocol produced a significant change in RMT, CSP, or MEP amplitude immediately after or $60 \mathrm{~min}$ after LF-rTMS. In addition, no LF-rTMS protocol produced an obvious trend.

\subsection{Dropouts and adverse effects}

All subjects had a normal neurological exam before and after TMS. Six subjects dropped out over the course of the study. Two subjects were lost to follow-up over the course of the several-month study duration. Four subjects experienced intolerant adverse effects that caused them to drop out of the study. In two of these subjects, the adverse effects were reported at the TMS session. One subject was at the beginning of his second TMS session when he reported nausea during the single-pulse TMS testing that preceded LF-rTMS. The other subject reported discomfort from stimulations after completing the single-pulse TMS testing immediately after LF-rTMS. In two subjects, adverse effect reporting was delayed by days to weeks. Two days after the first TMS session with protocol A, one subject reported difficulty concentrating and feeling dizzy for $24 \mathrm{~h}$. The other subject was contacted two weeks after her third TMS session (protocol B) and reported headaches ever since that session ended.

The two common adverse effects did not cause subjects to exit the study. The most frequent complaint was discomfort from the tight-fitting swimming cap. The second most common complaint was transient neck stiffness due to muscle strain from holding their heads still throughout TMS testing.

\section{Discussion}

Numerous investigators have explored the therapeutic potential of LF-rTMS in epilepsy and other brain disorders, yet the optimal LF-rTMS parameters for reducing cortical excitability remain unknown. Published studies have assessed the effects of LF-rTMS on cortical excitability and inhibition. Most directed LF-rTMS at primary motor cortex and measured effects on ipsilateral motor cortex excitatory and inhibitory tone by assessing RMT, CSP, MEP amplitude, intracortical inhibition (ICI), and/or intracortical facilitation (ICF). Each of these measurements has been reported to be affected by LFrTMS in some studies and unaffected by LF-rTMS in other studies. ${ }^{1}$ Even studies with strikingly similar LF-rTMS protocols have had different outcomes. ${ }^{7,17-19}$ Therefore, our findings of no LF-rTMS-induced change in MEP amplitude, RMT, or CSP align with findings in many other reports and supplement the understanding of the effect by assessing for changes in one systematic approach.

The LF-rTMS-induced change in cortical excitability that has been most likely to be reported is reduction in MEP amplitude, and this was not found in our study. In the review by Fitzgerald and colleagues, five of six studies reported no effect of subthreshold LF-rTMS on MEP amplitude, suggesting that LF-rTMS intensity may be important. ${ }^{1}$ However, in our study, there was no significant effect or trend from subthreshold or suprathreshold LF-rTMS on MEP amplitude.

In addition to LF-rTMS frequency and intensity, there are many other factors that can influence the modulation of cortical excitability by LF-rTMS, and these factors may explain why many LF-rTMS reports appear to disagree. ${ }^{20}$ These other 
factors include: train duration, pulse configuration, pulse duration, TMS coil shape/size, device and coil manufacturer, direction of induced current, and method for stabilizing the coil placement. ${ }^{21-26}$ Fixed factors within individual subjects also can influence the effect of LF-rTMS, and these include: skull-cortex distance, genetic factors, gender, and age. Finally, factors that fluctuate within individuals also can impact the modulatory effect of LF-rTMS, and these include: cognitive state, alertness, attention, hormones, circadian rhythms, and medications that act on the nervous system. Our systematic design was intended to minimize the impact of all of these factors and circumvent the debate raised by the discordant results in the published literature.

The lack of a significant change in our results does not necessarily indicate no effect of LF-rTMS on cortical excitability because a truly negative result requires sufficient statistical power to detect a small and relevant change. Chen and colleagues have proposed $0.38 \mathrm{mV}$ as the significant effect size for mean MEP amplitude with LF-rTMS using baseline MEP amplitude $1.95 \pm 1.63 \mathrm{mV}$ (mean \pm standard deviation) compared to $1.57 \pm 1.55 \mathrm{mV}$ after $0.9 \mathrm{~Hz}$ stimulation for $15 \mathrm{~min} .^{9}$ Using this result, a sample size of 140 subjects would be needed for $80 \%$ power. The MEP amplitude standard deviation in our results was $0.83 \mathrm{mV}$, which is less than the result found by Chen and colleagues, and 40 subjects would be needed for $80 \%$ power to affirm that the null hypothesis is true. Prior LF-rTMS studies have included 8-20 subjects, so our study contributes to the general understanding of LF-rTMS effect size and does not necessarily suggest an absence of effect. However, it may be useful when planning additional LF-rTMS studies because it demonstrates that the effect of the parameters we choose are not sufficient to produce a significant effect.

High intrinsic variability of cortical excitability measurements within individuals and multiple statistical testing each likely contributes to the positive outcomes identified in some publications. ${ }^{27,28}$ We found that no subject in this present study had a change in RMT or CSP with LF-rTMS that was outside of our previously published range of normal withinindividual variability. ${ }^{20}$ While cortical excitability variability can make it challenging to detect a statistically significant change, we did not appreciate a trend to suggest that a higher sample size might have brought statistical significance in this present report.

An interesting aspect of this study is a substantial dropout rate. One reason for dropouts is the long duration of this study, with some subjects lost to follow-up. Another significant issue was intolerable adverse effects, with nausea and discomfort causing two subjects to drop out during the middle of a TMS session. However, a sham stimulation exposure was not part of the protocol, so the adverse effects cannot be reliably attributed to the TMS. Similarly, the adverse effects that were present for an extended period as the participants were followed over months, including persisting headache and dizziness with difficulty concentrating, also cannot be reliably attributed to the TMS. Because of the possibility that the TMS produced these adverse effects and that the adverse effects impacted retention, future investigations comparing parameters would be best designed to include a sham stimulation group.

\section{Conclusion}

No LF-rTMS protocol produced a trend or significant change in MEP amplitude, RMT, or CSP. These findings are consistent with some of the other reports, while others have found LFrTMS-induced changes in these measures. The apparent discordance in the literature is likely due to the many factors that can affect the degree of excitability modulation induced by LF-rTMS, and the use of multiple statistical testing in much of the published TMS literature may have contributed to false positive findings. This study of four LF-rTMS protocols has employed a cross-over design and more limited variables and statistical analyses to avoid the limitations of prior investigations. Over the four-week duration, this study identified LF-rTMS adverse effects that were not present immediately after stimulation, including headache, dizziness, and difficulty in concentrating, and this also adds to the understanding of LF-rTMS and its potential as a therapeutic modality.

These results do not contradict results that LF-rTMS may reduce epileptic seizure frequency, as several groups have reported. ${ }^{7,29}$ The reported efficacy may be dependent on either a mechanism that the cortical excitability measurements do not detect or a change to cortical excitability that was not detected in our group. Furthermore, these results pertain to motor cortex only and LF-rTMS at the investigated settings may alter cortical excitability in other regions. An investigation that uses TMS with simultaneous EEG would be needed to determine this. ${ }^{30}$ However, the results of this investigation may be useful when designing future LF-rTMS experiments and treatment trials for epilepsy. Cortical excitability has been considered as a possible biomarker for epilepsy, so consideration of the impact of LF-rTMS on motor cortex excitability may still be worthwhile when choosing stimulation parameters in future studies. ${ }^{31}$ Investigations of LF-rTMS's effect on cortical excitability with a larger number of subjects and a control group are needed.

\section{Conflicts of interest}

All authors have none to declare.

\section{Acknowledgements}

For their generous support, the authors wish to thank the Brain Mapping Medical Center Research Organization, Brain Mapping Support Foundation, Pierson-Lovelace Foundation, The Ahmanson Foundation, Tamkin Foundation, Jennifer JonesSimon Foundation, Capital Group Companies Charitable Foundation, Robson Family, William M. and Linda R. Dietel Philanthropic Fund at the Northern Piedmont Community Foundation, Northstar Fund, and the National Center for Research Resources grants RR12169, RR13642, and RR08655. This work was also sponsored in part by a UCLA Stein Oppenheimer Award (JMS), the American Academy of Neurology Foundation (LMS), and the National EpiFellow Foundation (LMS). We greatly appreciate the statistical and methodological 
assistance of Jeffrey Gornbein, PhD, Robert M. Elashoff, $\mathrm{PhD}$, and Weiqing Liu from the UCLA Department of Biomathematics. Also, a special thanks to Sylvia Fong for help with manuscript preparation.

\section{REFERENCES}

1. Fitzgerald PB, Fountain S, Daskalakis ZJ. A comprehensive review of the effects of rTMS on motor cortical excitability and inhibition. Clin Neurophysiol. 2006;117:2584-2596.

2. Wassermann EM, Lisanby SH. Therapeutic application of repetitive transcranial magnetic stimulation: a review. Clin Neurophysiol. 2001;112:1367-1377.

3. Cincotta M, Borgheresi A, Gambetti C, et al. Suprathreshold $0.3 \mathrm{~Hz}$ repetitive TMS prolongs the cortical silent period: potential implications for therapeutic trials in epilepsy. Clin Neurophysiol. 2003;114:1827-1833.

4. Lefaucher J-P, Andre-Obadia N, Antal A, et al. Evidencebased guidelines on the therapeutic use of repetitive transcranial magnetic stimulation (rTMS). Clin Neurophysiol. 2014;125:2150-2206.

5. Kimiskidis VK, Kugiumtzis D, Papagiannopoulos S, Vlaikidis $\mathrm{N}$. Transcranial magnetic stimulation modulates epileptiform discharges in patients with frontal lobe epilepsy: a preliminary EEG-TMS study. Int J Neural Sys. 2013;23:1250035.

6. Brodbeck V, Thut G, Spinelli L, et al. Effects of repetitive transcranial magnetic stimulation on spike pattern and topography in patients with focal epilepsy. Brain Topogr. 2010;22:267-280

7. Kimiskidis VK, Valentin A, Kalviainen R. Transcranial magnetic stimulation for the diagnosis and treatment of epilepsy. Curr Opin Neurol. 2014;27:236-241.

8. Pascual-Leone A, Valls-Sole J, Wassermann EM, Hallett M. Responses to rapid-rate transcranial magnetic stimulation of the human motor cortex. Brain. 1994;117(Pt 4):847-858.

9. Chen R, Classen J, Gerloff C, et al. Depression of motor cortex excitability by low-frequency transcranial magnetic stimulation. Neurology. 1997;48:1398-1403.

10. Fierro B, Piazza A, Brighina F, et al. Modulation of intracortical inhibition induced by low- and high-frequency repetitive transcranial magnetic stimulation. Exp Brain Res. 2001;138:452-457.

11. Fitzgerald PB, Brown TL, Daskalakis ZJ, Chen R, Kulkarni J. Intensity-dependent effects of $1 \mathrm{~Hz}$ rTMS on human corticospinal excitability. Clin Neurophysiol. 2002;113:1136-1141.

12. Lang N, Harms J, Weyh $\mathrm{T}$, et al. Stimulus intensity and coil characteristics influence the efficacy of rTMS to suppress cortical excitability. Clin Neurophysiol. 2006;117:2292-2301.

13. Oldfield RC. The assessment and analysis of handedness: the Edinburgh inventory. Neuropsychologia. 1971;9:97-113.

14. Fritz C, Braune HJ, Pylatiuk C, Pohl M. Silent period following transcranial magnetic stimulation: a study of intra- and inter-examiner reliability. Electroencephalogr Clin Neurophysiol. 1997;105:235-240.

15. Boroojerdi B, Kopylev L, Battaglia F, et al. Reproducibility of intracortical inhibition and facilitation using the pairedpulse paradigm. Muscle Nerve. 2000;23:1594-1597.
16. Brasil-Neto JP, Cohen LG, Panizza M, et al. Optimal focal transcranial magnetic activation of the human motor cortex: effects of coil orientation, shape of the induced current pulse, and stimulus intensity. J Clin Neurophysiol. 1992;9:132-136.

17. Romero JR, Anschel D, Sparing R, Gangitano M, PascualLeone A. Subthreshold low frequency repetitive transcranial magnetic stimulation selectively decreases facilitation in the motor cortex. Clin Neurophysiol. 2002;113:101-107.

18. Modugno N, Curra A, Conte A, et al. Depressed intracortical inhibition after long trains of subthreshold repetitive magnetic stimuli at low frequency. Clin Neurophysiol. 2003;114:2416-2422.

19. Daskalakis ZJ, Moller B, Christensen BK, et al. The effects of repetitive transcranial magnetic stimulation on cortical inhibition in healthy human subjects. Exp Brain Res. 2006;174:403-412.

20. Pell GS, Roth Y, Zangen A. Modulation of cortical excitability induced by repetitive transcranial magnetic stimulation: influence of timing and geometrical parameters and underlying mechanisms. Prog Neurobiol. 2011;93:59-98.

21. Arai N, Okabe S, Furubayashi T, et al. Comparison between short train, monophasic and biphasic repetitive transcranial magnetic stimulation (rTMS) of the human motor cortex. Clin Neurophysiol. 2005;116:605-613.

22. Taylor JL, Loo CK. Stimulus waveform influences the efficacy of repetitive transcranial magnetic stimulation. J Affect Disord. 2007;97:271-276.

23. Kammer T, Beck S, Erb M, Grodd W. The influence of current direction on phosphene thresholds evoked by transcranial magnetic stimulation. Clin Neurophysiol. 2001;112:2015-2021.

24. Kammer T, Beck S, Thielscher A, Laubis-Herrmann U, Topka H. Motor thresholds in humans: a transcranial magnetic stimulation study comparing different pulse waveforms, current directions and stimulator types. Clin Neurophysiol. 2001;112:250-258.

25. Antal A, Kincses TZ, Nitsche MA, et al. Pulse configurationdependent effects of repetitive transcranial magnetic stimulation on visual perception. Neuroreport. 2002;13: 2229-2233.

26. Gugino LD, Romero JR, Aglio L, et al. Transcranial magnetic stimulation coregistered with MRI: a comparison of a guided versus blind stimulation technique and its effect on evoked compound muscle action potentials. Clin Neurophysiol. 2001;112:1781-1792.

27. Wassermann EM. Variation in the response to transcranial magnetic brain stimulation in the general population. Clin Neurophysiol. 2002;113:1165-1171.

28. Koski L, Schrader LM, Wu AD, Stern JM. Normative data on changes in transcranial magnetic stimulation measures over a ten hour period. Clin Neurophysiol. 2005;116:2099-2109.

29. Sun W, Mao W, Meng X, et al. Low-frequency repetitive transcranial magnetic stimulation for the treatment of refractory partial epilepsy: a controlled clinical study. Epilepsia. 2012;53:1782-1789.

30. Rotenberg A. Prospects for clinical applications of transcranial magnetic stimulation and real-time EEG in epilepsy. Brain Topogr. 2010;22:257-266.

31. Engel Jr J. Progress in epilepsy: reducing the treatment gap and the promise of biomarkers. Curr Opin Neurol. 2008;21:150-154. 